\title{
Abundance Ratios in Barium Stars
}

\author{
D.M. Allen ${ }^{1}$ and B. Barbuy ${ }^{1}$ \\ ${ }^{1}$ IAG-USP, Rua do Matão 1226, São Paulo, 05508-900, Brazil \\ email: dinah@astro.iag.usp.br
}

\begin{abstract}
Abundances of $\alpha$-, iron peak, s- and r-elements were determined for a sample of Barium stars and the $[\alpha$,iron peak $/ \mathrm{s}],[\alpha$,iron peak/r], $[\mathrm{s} / \mathrm{r}]$ ratios were derived.
\end{abstract}

Keywords. Stars: abundances, stars: AGB and post-AGB, nuclear reactions, nucleosynthesis, abundance

\section{Introduction}

Barium stars belong to a group of peculiar stars identified by Bidelman \& Keenan (1951). These stars are $\mathrm{G}$ and $\mathrm{K}$ giants showing strong lines of s-process elements, particularly Ba II and Sr II, as well as enhanced CH, CN and $\mathrm{C}_{2}$ bands. The discovery that HR 107, a dwarf star, shows composition similar to that of a mild Barium giant (Tomkin et al. (1989)) has pushed the search for new Barium dwarfs.

The temperatures of the sample stars determined from photometric data are in the range $4300 \mathrm{~K}<\mathrm{T}_{\text {eff }}<6500 \mathrm{~K}$. The metallicities derived from Fe I and Fe II lines are in the range $-1.2<[\mathrm{Fe} / \mathrm{H}]<0.0$, and gravities are $1.4<\log \mathrm{g}<4.6$, indicating that the sample includes giants, subgiants and dwarfs.

High-resolution spectra were obtained with the FEROS spectrograph at the ESO$1.5 \mathrm{~m}$ Telescope. The photometric data were obtained with Fotrap at the Zeiss telescope at LNA (Laboratório Nacional de Astrofísica-Brazil), as well as from the Hipparcos Catalogue, 2MASS (2 Micron All Sky Survey), and The General Catalogue Photometric Data (Geneva system).

\section{Abundance Ratios}

The abundances were determinated by spectrum synthesis of individual lines, using the code described by Barbuy et al. (2003).

The results of $[\mathrm{X} / \mathrm{Fe}]$ obtained for $\mathrm{Al}, \mathrm{Na}, \alpha$ - and iron peak elements are compatible with the values of $[\mathrm{X} / \mathrm{Fe}]$ presented in the literature for normal disk stars in the same range of metallicities.

For $\mathrm{Na}, \mathrm{Al}, \alpha$ and iron peak elements, the relation between $[\mathrm{X} / \mathrm{Fe}]$ and $[\mathrm{Fe} / \mathrm{H}]$ is approximately constant in the small range of metallicities of the sample stars. On the other hand, the heavy elements show a higher dispersion which can be explained by the different amounts of enriched material that each star received from its evolved companion. The results for overabundances in s-elements indicate that there is no significant dependence on the luminosity class.

The excesses of $\mathrm{Na}, \mathrm{Al}, \alpha$ and iron peak elements relative to Ba show a decreasing trend with $[\mathrm{Ba} / \mathrm{H}]$. Relations between $[\mathrm{X} / \mathrm{Ba}]$ and $[\mathrm{Fe} / \mathrm{H}]$ show an increasing trend. Considering that $\mathrm{Ba}$ represents s-process elements, one can consider that these relations describe the relations between s-process and other nucleosynthetic processes. Regarding relations involving the r-process element $\mathrm{Eu}$, there is a range of $[\mathrm{Eu} / \mathrm{H}]$ where $[\mathrm{X} / \mathrm{Eu}]$ is essentially 
constant and shows a decreasing trend at the metal-poor end. However, the dispersion does not allow a definitive conclusion. For most stars, $[\mathrm{X} / \mathrm{Eu}]<0$, except for $\mathrm{Mg}$, Co and Sc. $[\mathrm{X} / \mathrm{Eu}] \mathrm{x}[\mathrm{Fe} / \mathrm{H}]$ seems to be constant, with the exception of $[\mathrm{Cr} / \mathrm{Eu}]$. The dispersion comes from the fact that stars of different masses produce different amounts of each element. Furthermore, r-elements are not produced in SN Ia, in contrast to most of the other elements. Given that the main component of s-process takes place in a different site (AGB stars), the relation between those elements and $\mathrm{Ba}$ does not follow the same pattern. We also conclude that there is no significant dependence of abundances ratios on the luminosity class.

The relations between s- and r-elements for barium stars and post-AGB stars from Reyniers et al. (2004) and van Winckel \& Reyniers (2000) were used for comparison purposes. The pattern of $[\mathrm{X} / \mathrm{Eu}, \mathrm{Ba}]$ versus $[\mathrm{Fe} / \mathrm{H}]$ for barium stars is similar to postAGBs. The dispersion in $[\mathrm{Sr} / \mathrm{Ba}] \mathrm{x}[\mathrm{Fe} / \mathrm{H}]$ for barium and AGB stars is similar to normal stars as given in Burris et al. (2000), suggesting that the abundance of elements relative to the weak component of the s-process is the same for normal, post-AGB and barium stars, and the yield relative to the main component is similar for light and heavy s-elements, keeping the same dispersion of normal stars.

\section{3. s-Process Indexes}

The ratios $[\mathrm{s} / \mathrm{Fe}],[\mathrm{ls} / \mathrm{Fe}],[\mathrm{hs} / \mathrm{Fe}]$ and $[\mathrm{hs} / \mathrm{ls}]$ were determined. We considered for the ls index the light s-elements Sr, Y and Zr, for the hs index the heavy s-elements Ba, La, $\mathrm{Ce}$ and $\mathrm{Nd}$ and for the s index the light and heavy s-elements.

The results were compared to those from Junqueira \& Pereira (2001), Luck \& Bond (1991) and North et al. (1994). [s/Fe] and [hs/ls] show a decreasing trend for $[\mathrm{Fe} / \mathrm{H}]>$ -1 . However, there is no well-defined trend for $[\mathrm{ls} / \mathrm{Fe}]$ and $[\mathrm{hs} / \mathrm{Fe}]$ as a function of $[\mathrm{Fe} / \mathrm{H}]$.

\section{Acknowledgements}

DMA acknowledges a FAPESP fellowship $n^{\circ}$ 00/10405-8. The FEROS observations at the European Southern Observatory (ESO) were carried out within the Observatório Nacional ON/ESO and ON/IAG agreements, under Fapesp project $n^{\circ}$ 1998/10138-8. BB acknowledges grants from CNPq and FAPESP.

\section{References}

Barbuy B., Perrin, M.-N., Katz, D., Coelho, P., Cayrel, R., Spite, M., \& Van’t Veer-Menneret, C. 2003, A\&A 404, 661

Bidelman W.P. \& Keenan P.C. 1951, ApJ 114, 473

Burris, D.L., Pilachowski C.A., Armandroff T.E., Sneden C., Cowan J.J., \& Roe H. 2000, ApJ 544,302

Junqueira S. \& Pereira C.B. 2001, AJ 122, 360

Luck R.E. \& Bond H.E. 1991, ApJS 77, 515

North P., Berthet S., \& Lanz T. 1994, A\&A 281, 775

Reyniers M., Van Winckel H., Gallino R., \& Straniero O. 2004, A $\& A$ 417, 269

Tomkin J., Lambert D.L., Edvardsson B., Gustafsson B., \& Nissen P.E. 1989, A\&GA 219, L15

van Winckel, H. \& Reyniers M. 2000, A\&\&A 354, 135 